\title{
Precipitation recycling in the Amazon basin
}

\author{
By E. A. B. ELTAHIR and R. L. BRAS* \\ Massachusetts Institute of Technology, USA
}

(Received 1 June 1993; revised 26 November 1993)

SUMMARY

Precipitation recycling is the contribution of evaporation within a region to precipitation in that same region. The recycling rate is a diagnostic measure of the potential for interactions between land surface hydrology and regional climate. In this paper we present a model for describing the seasonal and spatial variability of the recycling process. The precipitation recycling ratio, $\rho$, is the basic variable in describing the recycling process. $\rho$ is the fraction of precipitation at a certain location and time which is contributed by evaporation within the region under study. The recycling model is applied in studying the hydrologic cycle in the Amazon basin. It is estimated that about $25 \%$ of all the rain that falls in the Amazon basin is contributed by evaporation within the basin. This estimate is based on analysis of a data set supplied by the European Centre for Medium-range Weather Forecasts. The same analysis is repeated using a different data set from the Geophysical Fluid Dynamics Laboratory. Based on this data set, the recycling ratio is estimated to be $35 \%$. The seasonal variability of the recycling ratio is small compared with the yearly average. The new estimates of the recycling ratio are compared with results of previous studies, and the differences are explained.

\section{INTRODUCTION}

Precipitation recycling is the contribution of evaporation within a region to precipitation in that same region. The studied region can be part of the ocean, the land, or it may contain both ocean and land surfaces. The estimate of precipitation recycling for a continental land mass provides a measure of the potential for interactions between land surface hydrology and regional climate. A high estimate of precipitation recycling is not sufficient to conclude a strong role for land surface hydrology in the regional climate. It rather suggests a strong potential for that role; by strong role we mean that significant changes in surface hydrology necessarily result in significant impacts on the regional climate. On the other hand, a low estimate of precipitation recycling implies that the only possible role of land surface hydrology is through the effects of vegetation on surface albedo. Hence, studying precipitation recycling of land areas provides useful information on the possible interactions of hydrology and climate.

The recycling study described in this paper is part of a larger effort which focuses on the interactions of land surface hydrology and regional climate of the Amazon basin. The high rates of deforestation in the Amazon basin have prompted wide concern about the possible impacts of deforestation on climate. Previous studies, for example Dickinson and Henderson-Sellers (1988), Lean and Warrilow (1989) and Shukla et al. (1990), focused on the impacts on regional climate due to deforestation of the Amazon rain forest using a modelling approach. In this paper, a precipitation recycling model is developed for studying the role of hydrology in the Amazon climate.

Early studies of precipitation recycling focused on the continental land mass of North America. While McNish (1936) and Horton (1943) suggest that the contribution of evaporation from a land region to precipitation in the same region is significant, the studies of Holzman (1937), Benton et al. (1950) and McDonald (1962) criticize these views and emphasize the large mobility of atmospheric air masses and the important role of rainfall-producing mechanisms. Most of the above studies involved limited data analysis and little theoretical description of the recycling process.

\footnotetext{
* Corresponding author: Ralph M. Parsons Laboratory, Department of Civil and Environmental Engineering, Massachusetts Institute of Technology, Cambridge, MA 02139, USA.
} 
Budyko (1974) developed a model for estimating recycling in large regions. Budyko's model describes the process along a single streamline and provides a lumped estimate of recycling. Budyko (1974) used the model to estimate recycling in the European part of the USSR and estimated that the contribution of recycled precipitation is about $10 \%$. In a recent study Brubaker et al. (1991) modified the Budyko model to account for the fact that the spatial orientation of the region under study may not lie parallel to a streamline. The modified model is used in studying recycling in different regions of the world, including the Amazon basin. It is estimated that recycling in the Amazon varies in the range from 15 to $30 \%$ in the different months.

Many previous studies provide estimates for precipitation recycling in the Amazon basin. Molion (1975) estimated that recycled precipitation accounts for about $56 \%$ of the total precipitation. This estimate is the ratio of total evaporation to total precipitation in the basin. The accuracy of this estimate will be discussed in section 7. Lettau et al. (1979) presented a model of recycling based on their climatonomy theory. The model describes the recycling process along a single streamline. Lettau et al. (1979) estimated that the contribution of recycled precipitation varies from about $16 \%$ at the eastern sub-region to $47 \%$ at the western sub-region of the Amazon basin.

Isotopic analysis of the natural composition of water is an attractive procedure for identifying the sources of precipitation. Fractionation takes place when water undergoes condensation or evaporation. Naturally heavy isotopes favour the liquid phase. Owing to condensation, atmospheric water vapour is gradually depleted of heavy isotopes as it moves over the Amazon basin from east to west. Salati et al. (1979) analysed data of the $\mathrm{O}^{18}$ concentration from different stations in the Amazon basin and found that the inland gradient is smaller than the gradients observed in Europe. They attributed this difference to the significant contribution of local evaporation to atmospheric water vapour. The isotopic data collected in the Amazon basin are inhomogenous, which may indicate significant spatial and seasonal variability in the recycling process. A comparison between the isotopic composition of the advected vapour flux and the isotopic composition of the river discharge indicates a significant flux of water vapour out of the basin. Although isotopic analysis provides useful information about the recycling process, it is difficult to make quantitative estimates of recycling based solely on isotopic data. That is why the study of Salati et al. (1979) does not provide a quantitative estimate of recycling in the Amazon basin. Models of precipitation recycling which utilize water vapour flux and evaporation data, for example the models of Budyko (1974) and Lettau et al. (1979), provide these quantitative estimates.

Here we present a model of precipitation recycling which describes both the spatial and the seasonal variability of the recycling process. The model involves only two assumptions. It assumes that atmospheric water vapour is well mixed and that the rate of change of storage of water vapour is negligible compared with water vapour fluxes at the time-scale for which the model is applicable. Observational evidence which indicates that both assumptions are reasonable idealizations of the real world is discussed.

The model is applied in estimation of recycling in the Amazon basin using two different data sets. The main results of the study are obtained by applying the recycling model to the European Centre for Medium-range Weather Forecasts (ECMWF) data. In order to verify the main results of the study, the same model is also applied to the Geophysical Fluid Dynamics Laboratory (GFDL) data to obtain a different estimate of recycling. In the following sections the results obtained using the ECMWF data will be described in some detail. The corresponding results which are based on the GFDL data are described briefly. The conclusions of the study are based on the two estimates.

The paper is organized in eight sections. Section 2 outlines the development of the 
new recycling model. The details of the estimation procedure which describes how the model can be applied using any data set are presented in section 3. The ECMWF data are described in section 4. The application of the model to the Amazon basin using the ECMWF data and GFDL data are described in sections 5 and 6 respectively. Section 5 includes estimates of the seasonal and spatial distributions of precipitation recycling. Section 7 explains why previous studies overestimated precipitation recycling in the Amazon basin. Section 8 includes a discussion and the conclusions of this study.

\section{Precipitation RECYCling MODEL}

In developing this model we consider two species of water vapour molecules: those which evaporate outside the region and those which evaporate within the region. The definition of the word 'region' includes all the area under study, e.g. the Amazon basin. It is not restricted to the area of a single grid point. For a finite control volume of the atmosphere located at any point within the region, conservation of mass requires the following relations:

$$
\left.\begin{array}{c}
\frac{\partial N_{\mathrm{w}}}{\partial t}=I_{\mathrm{w}}+E-O_{\mathrm{w}}-P_{\mathrm{w}} \\
\frac{\partial N_{\mathrm{o}}}{\partial t}=I_{\mathrm{o}}-O_{\mathrm{o}}-P_{\mathrm{o}}
\end{array}\right\}
$$

where subscripts $w$ and o denote molecules which evaporate within the region and outside the region respectively. $I$ and $O$ are inflow and outflow, $P$ is precipitation, and $E$ is evaporation (see Fig. 1). $N, I, O, P$ and $E$ are variables in space and time. Each of $I$ and $O$ is a summation of the components of flux in the two horizontal directions.

It is observed that water vapour is well mixed in the planetary boundary layer (PBL) of the earth's atmosphere. The depth of the PBL is in the order of one kilometre and contains most of the water vapour in the atmosphere. Observations of the vertical distribution of water vapour and other conserved tracers show a practically uniform

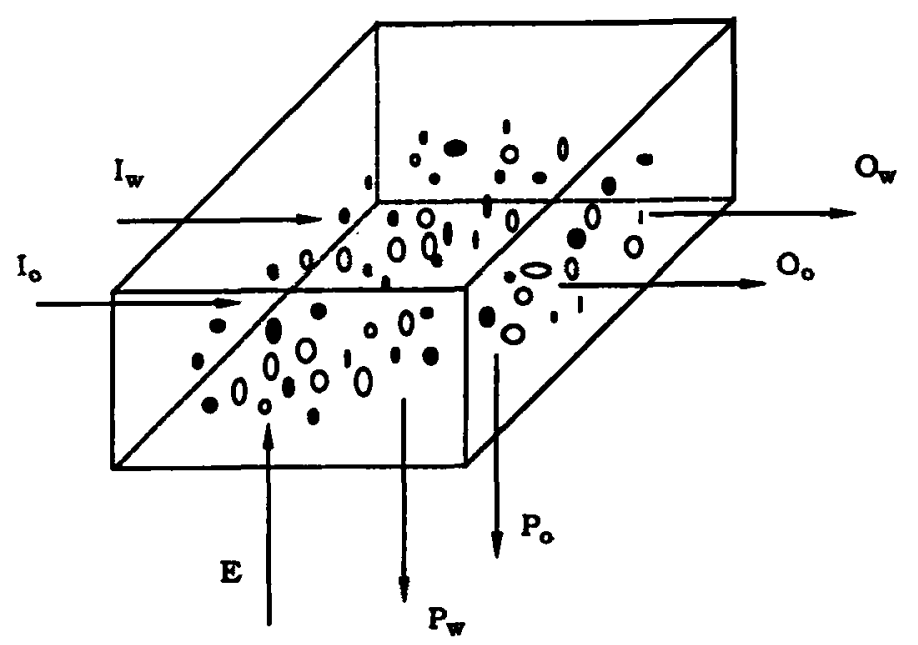

Figure 1. Schematic of the atmospheric control volume (see text for explanation of symbols). 
distribution through the PBL up to the level where the air from the PBL mixes with the upper air. For observations in mid-latitudes see Crum and Stull (1987). For observations from the Amazon region see Harris et al. (1988) and companion papers describing the Amazon Boundary Layer Experiment. Mixing of water vapour in the PBL is primarily achieved by dry thermal convection. This process is very efficient in mixing of water vapour and other pollutants. Lamb (1982) compiled the results of many field experiments, studying air pollutants which are released at the surface, the data indicate that mixing in the PBL occurs at very short time-scales. It takes about 15 minutes for water vapour molecules evaporating at the surface to mix in the vertical for up to a depth of one kilometre. Above the boundary layer it is frequently observed that the air forming clouds mixes with the surrounding environment (see Paluch (1979)). Based on these observations we make the assumption that water vapour molecules from the two species defined above are well mixed, which implies that

$$
\rho=\frac{P_{\mathrm{w}}}{\left(P_{\mathrm{w}}+P_{\mathrm{o}}\right)}=\frac{O_{\mathrm{w}}}{\left(O_{\mathrm{w}}+O_{\mathrm{o}}\right)}=\frac{N_{\mathrm{w}}}{\left(N_{\mathrm{w}}+N_{\mathrm{o}}\right)}
$$

where $\rho$ is defined as the precipitation recycling ratio. At any location within the region, $\rho$ estimates the ratio of recycled precipitation to the total precipitation falling at that location. Recycled precipitation consists of molecules of water vapour which were in the atmosphere as a result of an evaporation event at that location, or elsewhere in the region under study.

Figure 2 shows a comparison between the monthly outflow of water vapour, evaporation and the change in storage of water vapour at a single location in the Amazon basin. It is evident that at the monthly time-scale, the rate of change of storage of water vapour is very small compared with the atmospheric water vapour fluxes. This observation

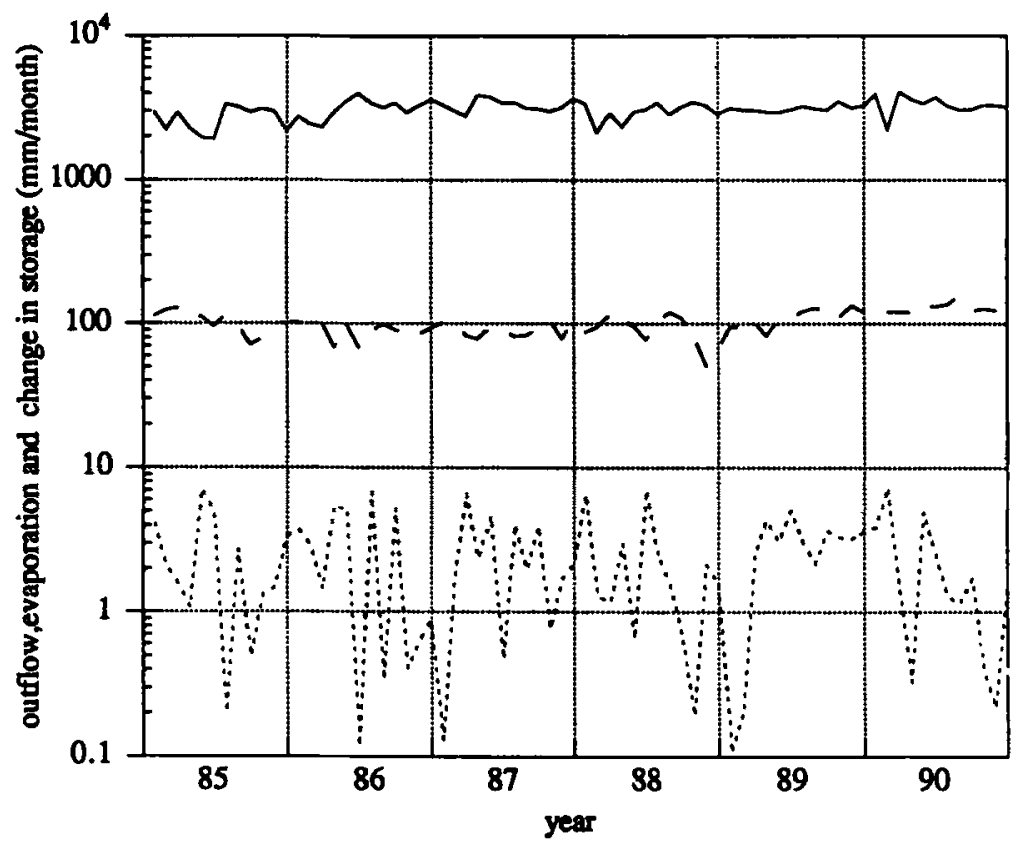

Figure 2. Comparison between atmospheric fluxes of water vapour and rate of change in water vapour storage at a grid point in the Amazon basin, from the ECMWF data set. Full line, outflow; dashed line, evaporation; and dotted line, change in storage. 
combined with the well mixing assumption suggest that change of storage of any of the two species, $N_{\mathrm{w}}$ or $N_{\mathrm{o}}$, is small compared with its flux (see appendix). We then make the assumption that the derivatives in Eq. (1) are zero. Rearranging Eq. (1) we get

$$
\left.\begin{array}{c}
I_{\mathrm{w}}+E=O_{\mathrm{w}}+P_{\mathrm{w}} \\
I_{\mathrm{o}}=O_{\mathrm{o}}+P_{\mathrm{o}}
\end{array}\right\}
$$

substituting for $O_{\mathrm{w}}, P_{\mathrm{w}}, O_{\mathrm{o}}$ and $P_{\mathrm{o}}$ from Eq. (2) into Eqs. (3)

$$
\begin{gathered}
I_{\mathrm{w}}+E=\rho\left(O_{\mathrm{w}}+O_{\mathrm{o}}\right)+\rho\left(P_{\mathrm{w}}+P_{\mathrm{o}}\right) \\
I_{\mathrm{o}}=(1-\rho)\left(O_{\mathrm{w}}+O_{\mathrm{o}}\right)+(1-\rho)\left(P_{\mathrm{w}}+P_{\mathrm{o}}\right) .
\end{gathered}
$$

By dividing Eq. (4a) by Eq. (4b), and rearranging, we get

$$
\rho=\frac{\left(I_{\mathrm{w}}+E\right)}{\left(I_{\mathrm{w}}+E+I_{\mathrm{o}}\right)}
$$

Equation (5) is the formula for estimating recycling in this model.

The spatial resolution of the data should be small enough to resolve significant spatial variability in evaporation and fluxes. The temporal resolution should be large enough for the above assumption of negligible change in storage to hold. It should also be large compared with the travel time across the region studied.

In the following we compare the new recycling model with the models of Budyko (1974) and Lettau et al. (1979). The new model describes the spatial variability of the process in two dimensions, while Budyko's model provides a lumped estimate of recycling. The Lettau model describes the variability of the process in one dimension along a single streamline. Further, the new model does not make assumptions about the spatial distribution of evaporation and precipitation, while Budyko's model assumes a uniform distribution for evaporation and precipitation. The Lettau model involves a linearity assumption in relating precipitation to evaporation and precipitable water. Hence, the recycling model introduced in this paper achieves more in terms of the spatial distribution of the recycling process, but makes fewer or milder assumptions.

\section{ESTIMATION PROCEDURE}

The estimation procedure suggested with the new recycling model consists of the following steps which are illustrated by Fig. 3.

1. The flux and evaporation data are interpolated in a rectangular grid which covers the total area of the region. $\rho$ is estimated at points which lie at half the distance between the grid points.

2. The estimation procedure is a trial and error technique where $\rho$ is first guessed at all points in the data grid. Equation (2) is then used in partitioning the flux at every point and in both directions into $O_{\mathrm{w}}$ and $O_{\mathrm{o}}$. It is important to note that $O_{w}$ and $O_{\mathrm{o}}$ for one grid point are $I_{\mathrm{w}}$ and $I_{\mathrm{o}}$ for an adjacent point. These estimates of $I_{\mathrm{w}}$ and $I_{\mathrm{o}}$ together with $E$ are then used in Eq. (5) to arrive at improved estimates of the distribution of $\rho$. Following this procedure, $\rho$ is estimated downstream for all the points. The new estimates of $\rho$ are then compared with the previous estimates. The procedure is repeated until the new and previous estimates of $\rho$ converge. The estimation procedure is found to converge very quickly and to be insensitive to the initial guess of $\rho$.

3. The recycling ratio for the total area of the basin, $\rho_{t}$, and for any month, $k$, is obtained by 


$$
\rho_{\mathrm{t}}(k)=\sum_{i, j} p(i, j, k) \rho(i, j, k)
$$

where $p(i, j, k)$ is precipitation at the grid box $(i, j)$, and month $k$ is normalized by the total precipitation in the basin for that month.

4. Similarly the yearly estimate of $\rho$ at any grid point $(i, j)$ is estimated by

$$
\rho_{y}(i, j)=\sum_{k} p(i, j, k) \rho(i, j, k)
$$

where $p(i, j, k)$ is defined this time as the precipitation at the grid box $(i, j)$, and month $k$ is normalized by the yearly precipitation at $(i, j)$.

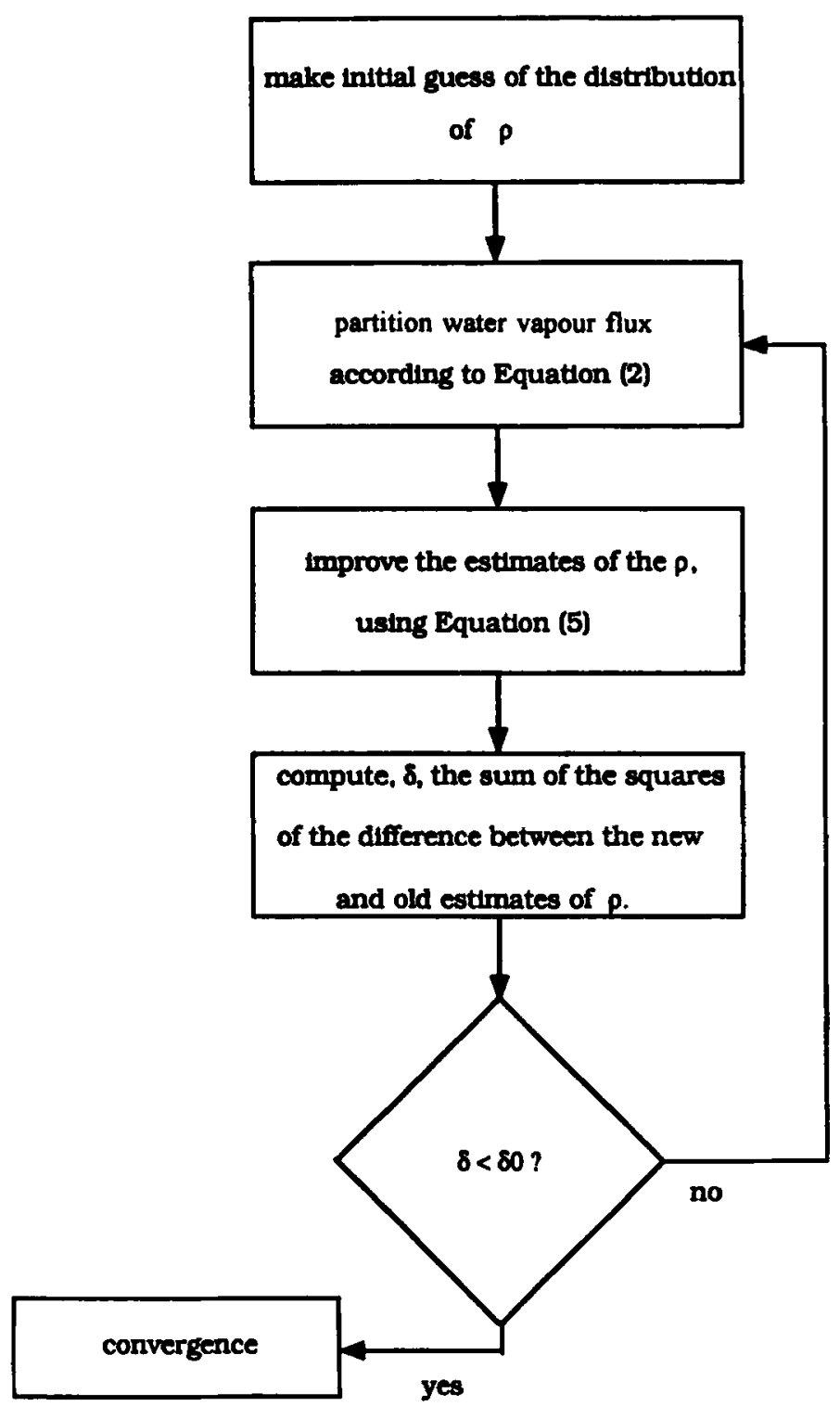

Figure 3. Flow chart of the estimation procedure. $\rho$ is the precipitation recycling ratio. 


\section{ECMWF DATA}

The data used in this study are a sub-set of the ECMWF global data. The sub-set consists of temperature, relative humidity, and wind at 14 pressure levels $(1000,850$, $700,500,400,300,250,200,150,100,70,50,30$, and $10 \mathrm{mb})$. It also includes evaporation data. The evaporation data are accumulated amounts over six hours and available every six hours. The rest of the data are provided twice daily. The data cover the period 1985-90 inclusive and have a spatial resolution of $2.5^{\circ}$ latitude by $2.5^{\circ}$ longitude. Trenberth and Olson (1988) point out that the ECMWF analyses are believed to be the best operational global analyses available for general use.

The data assimilation system at the ECMWF combines data from surface meteorological stations, upper-air observations and satellite data. The radiosonde network in the region consists of about ten stations which are located within $2000 \mathrm{~km}$ of the centre of the Amazon basin. These stations provide daily measurements. There are about 25 surface stations in the region providing twice-daily measurements. The location of the surface and upper-air stations are shown on Fig. 4. It is clear that the frequency and spatial coverage of conventional observations in the Amazon basin are limited. Under these conditions, the final output from any data assimilation procedure may reflect some of the model bias. Other possible sources of error are the effects of model resolution on the representation of orography, and the interpolation from model vertical levels to the standard pressure levels. Illari (1989) studied the quality of the ECMWF humidity analysis and showed that, in the tropics, the data obtained from radiosondes, satellites and from the model's first-guess are similar in terms of quality and informational content. This is particularly important since it shows that the coverage of the tropics by satellite data supplements significantly the sparse radiosonde data.

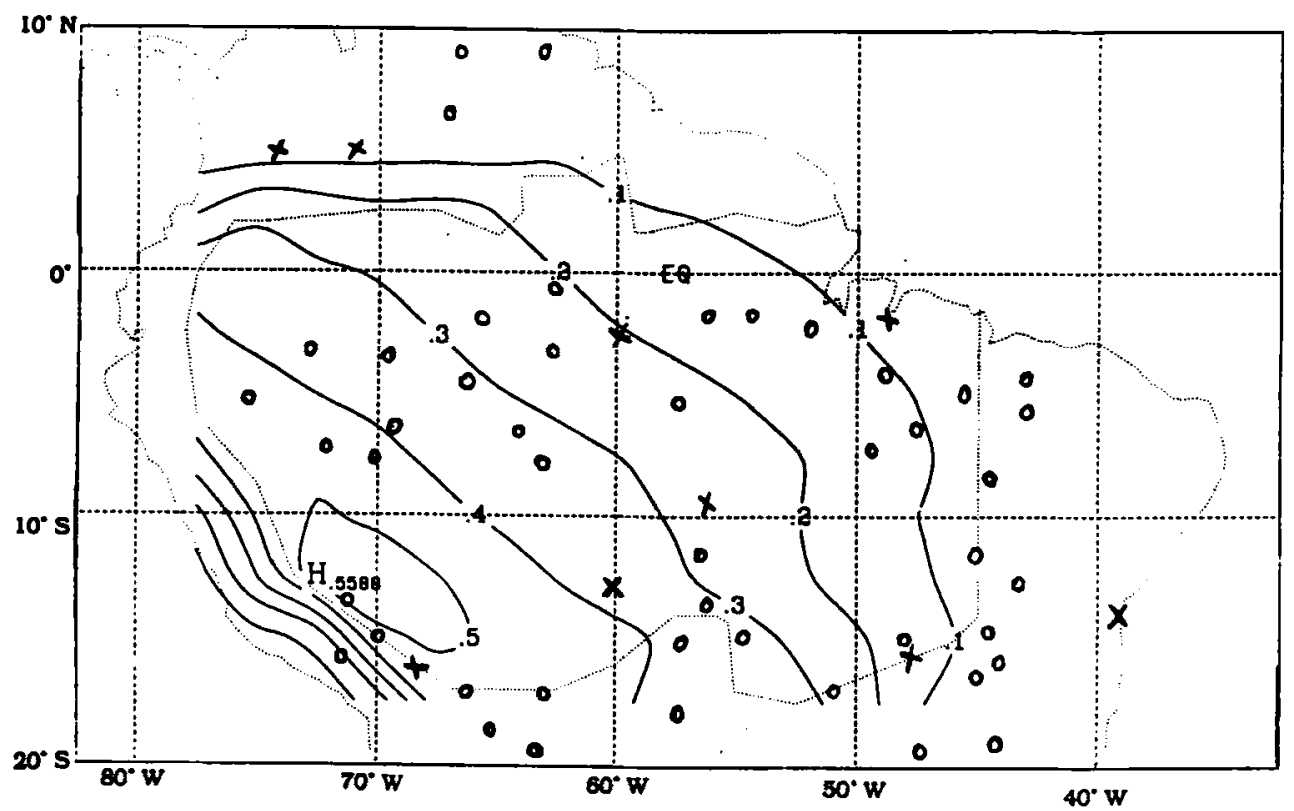

Figure 4. Spatial distribution of the annual precipitation recycling ratio in the Amazon basin. The boundaries of the Amazon basin are shown with a dotted line. Also shown are the locations of the observation stations which are the source of the data used by the ECMWF in their assimilation procedure. $A$ circle indicates a surface station, and a cross indicates a station with upper-air observations. The number and locations of these stations are approximate, they are based on information provided by the ECMWF. 
The ECMWF data set includes estimates of surface evaporation. These estimates are calculated by a surface hydrology scheme, using surface parameters and atmospheric variables. Surface hydrology is modelled using a simple 'bucket' hydrology representation, and evaporation is computed using a similarity formulation. In order to validate the evaporation data, we compare the ECMWF data with the observations of Shuttleworth (1988). Figure 5 shows the average monthly evaporation computed from the ECMWF data for six years at the grid-cell which covers Manaus in the Amazon basin. It also shows the estimates of monthly evaporation at a single site near Manaus averaged from two years of the Amazon Region Micro-meteorological Experiment. The two data sets are in reasonable agreement, the difference between the estimates of yearly evaporation is in the order of $5 \%$.

The atmospheric water vapour flux is computed from the data on wind, temperature, and humidity. By making a hydrostatic assumption and utilizing the relation between water vapour mixing ratio and vapour pressure, we derive the following formula for computing flux

$$
F_{i}=\frac{\varepsilon L_{i}}{g} \sum_{n} R H_{n} \cdot e_{\mathrm{s}}\left(T_{n}\right) \cdot U_{i n} \cdot \mathrm{d} \ln P_{n}
$$

where $i=1,2$ corresponds to the zonal and meridional directions respectively. $n$ indicates the pressure level. $\varepsilon$ is the ratio of the molecular weight of water vapour to that of dry air and $g$ is the gravitational acceleration. $L$ is the linear scale perpendicular to the flux direction. $R H$ is relative humidity and $e_{\mathrm{s}}$ is saturation vapour pressure. $T$ is temperature, $U$ is wind speed and $P$ is pressure. The above formula is used in computing the flux at each grid point. The monthly fluxes and evaporation are estimated by integrating the corresponding data for each month.

The ECMWF data set does not include precipitation. Precipitation is estimated from

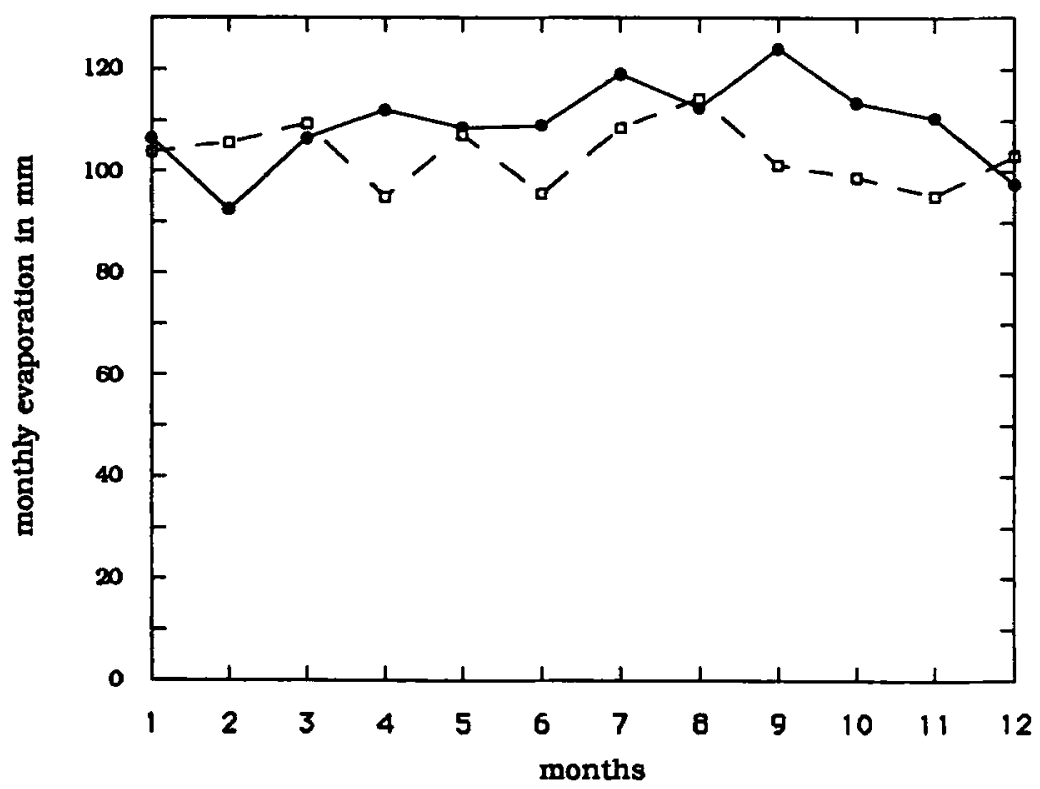

Figure 5. Comparison of the evaporation estimates for Manaus, from the ECMWF data set ( $\square--\square)$ and from the ARME (O) 
the water balance of the atmospheric column above each grid point. Since the change in storage of water vapour is small compared with the fluxes, as illustrated by Fig. 3, the continuity equation for each grid box is reduced to

$$
P=E-\nabla \cdot \mathbf{F} \text {. }
$$

The estimates of precipitation by this method are less accurate than the estimates of flux and evaporation. The noise in those estimates is reduced by averaging the data for each month of the year from the six-years' data. The estimates of monthly precipitation are used as the weighting factors in Eqs. (6) and (7).

\section{ESTIMATION OF PRECIPITATION RECYCLING USING THE ECMWF DATA SET}

The model described in the previous sections is used to estimate recycling in the Amazon basin. The estimation procedure outlined in section 3 is applied using the evaporation, flux and precipitation data. Figure 4 is the annual recycling map for the Amazon basin. It shows that the contribution of recycling increases westward and southward. The maximum rate of recycling occurs at the south-western corner of the basin. At that corner more than $50 \%$ of the precipitation is contributed by evaporation within the Amazon basin. In order to compute a lumped estimate of the annual precipitation recycling ratio in the Amazon basin, the annual recycling map is averaged by a weighting procedure similar to that of Eq. (6). The only difference being the use of annual precipitation instead of monthly precipitation. It is estimated that $25 \%$ of all the rain which falls in the Amazon basin is contributed by evaporation within the basin.

The recycling maps for some months of the year are shown in Fig. 6. The spatial distribution of recycling in the Amazon basin has significantly different patterns in the different seasons. During most of the year the east-west gradient dominates the recycling map, consistent with the predominantly easterly winds in the basin. But during the southern hemisphere summer, especially in December, the south-north gradient is larger, consistent with the southward migration of the intertropical convergence zone and the resulting northerly flux over most of the Amazon basin. It is revealing to compare the recycling maps for the months of Jume and December with the corresponding flux maps in Fig. 7. The recycling model is capable of describing the changes in the recycling patterns which result from the seasonal variability of the water vapour flux patterns.

Another important feature is the ability of the recycling model to reflect the effects of the spatial variability of evaporation in the recycling pattern. This is illustrated by comparing the evaporation maps for the months of June and August. Evaporation in August is significantly smaller than evaporation in June, particularly at the south-eastern sub-region, see Fig. 8. The August recycling map reflects this feature in the significant reduction of the recycling ratio over a large area.

The seasonal variability of $\rho_{\mathrm{t}}$ is estimated by applying Eq. (6) to the recycling map of each month. Figure 9 (a) shows $p_{t}$ for the different months. The seasonal variability in the recycling estimates is small compared with both the average and the accuracy of the estimates themselves.

Since the estimates of precipitation, from Eq. (9), are less accurate compared with the evaporation and flux data, the sensitivity of the recycling results is assessed by computing $\rho_{\mathrm{t}}$, assuming a uniform spatial distribution of precipitation. The results are shown in Fig. 9(b). A comparison of Fig. 9(a) with Fig. 9(b) suggests that the estimates of recycling are only slightly sensitive to the rainfall distribution. The differences between the recycling ratios in the two figures are within the error bars of the estimates (order of $10 \%)$. 

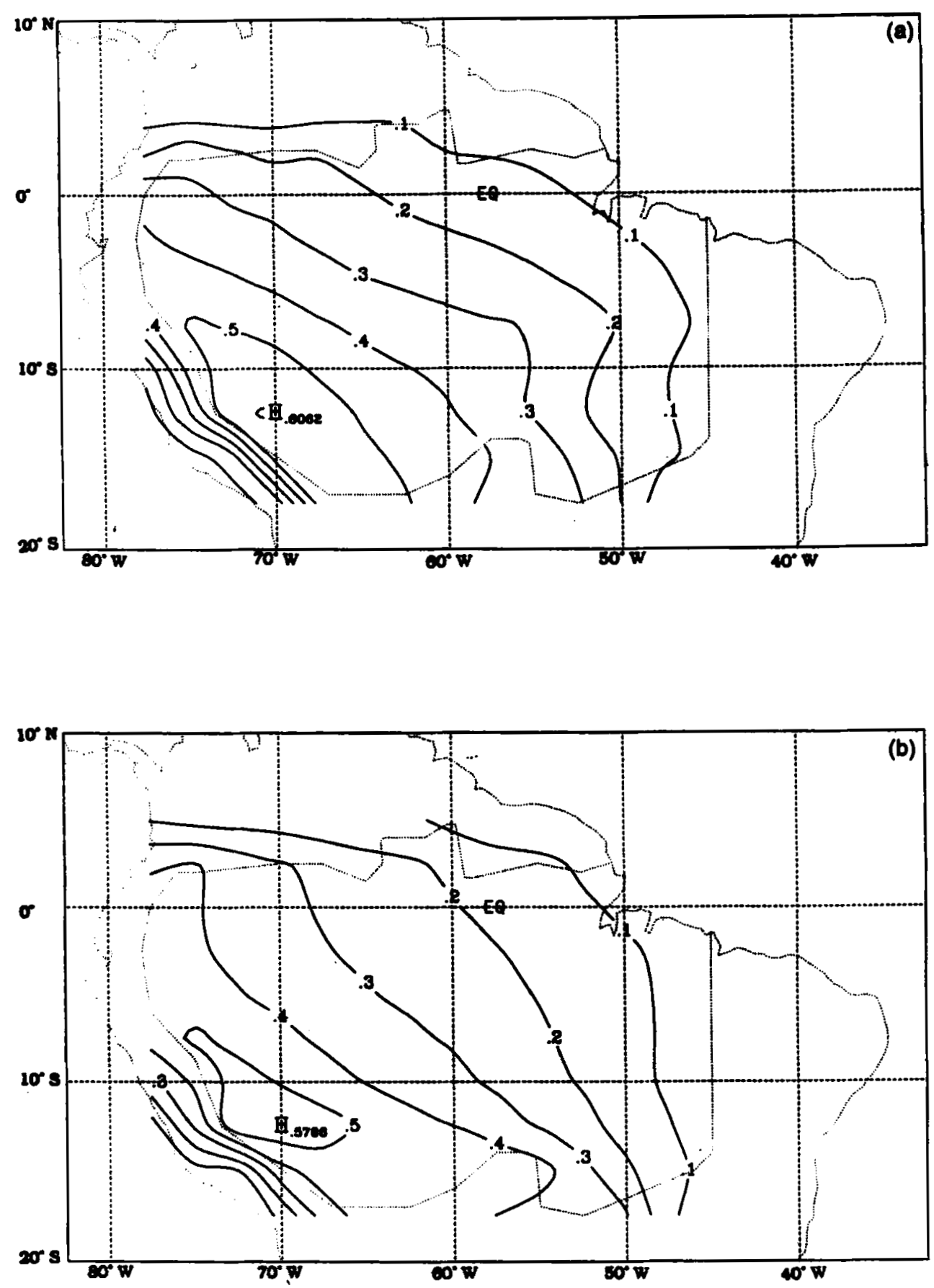

Figure 6. Spatial distribution of the precipitation recycling ratio for: (a) March, (b) June, (c) August and (d) December. 

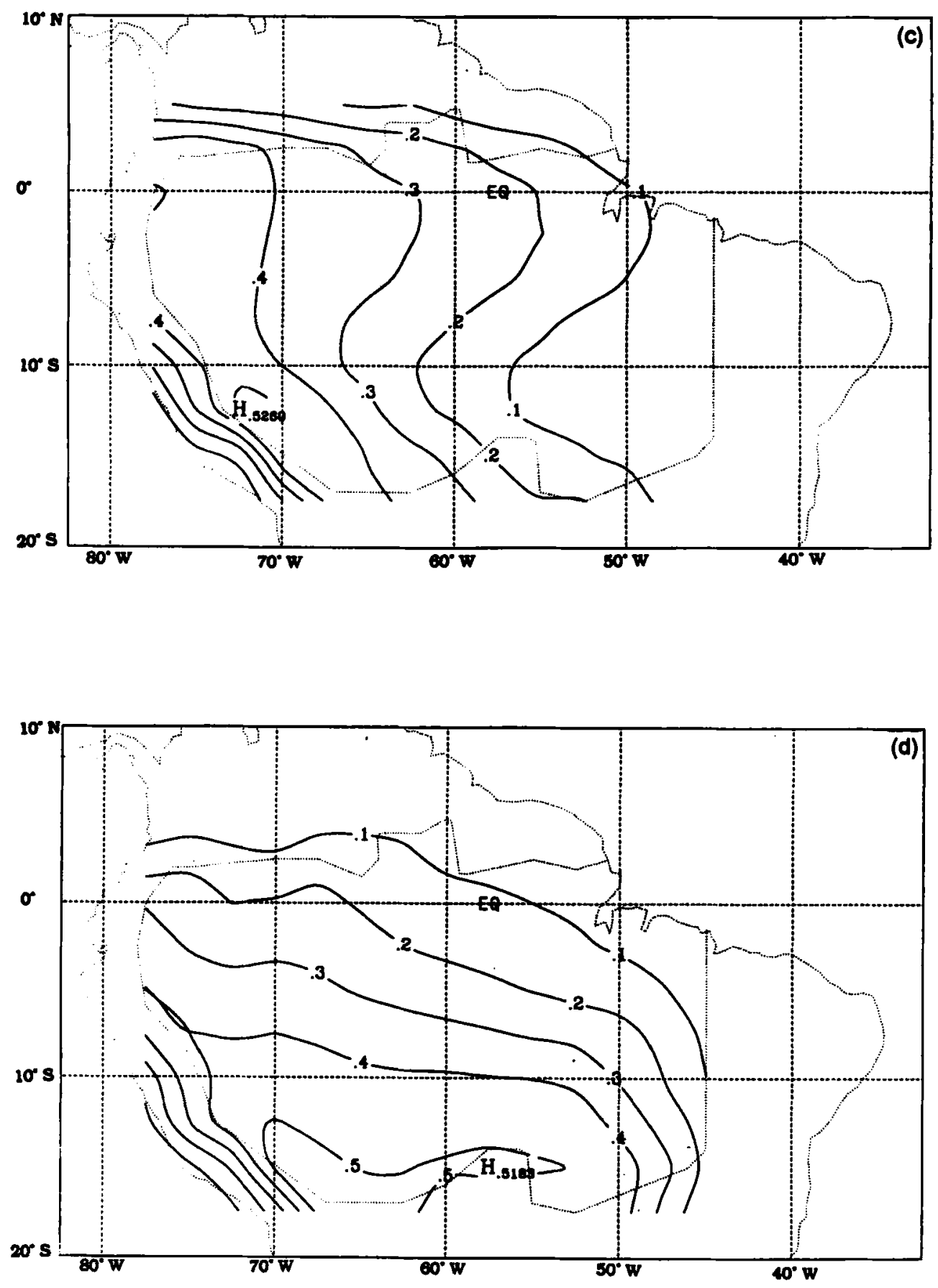

Figure 6. Continued. 

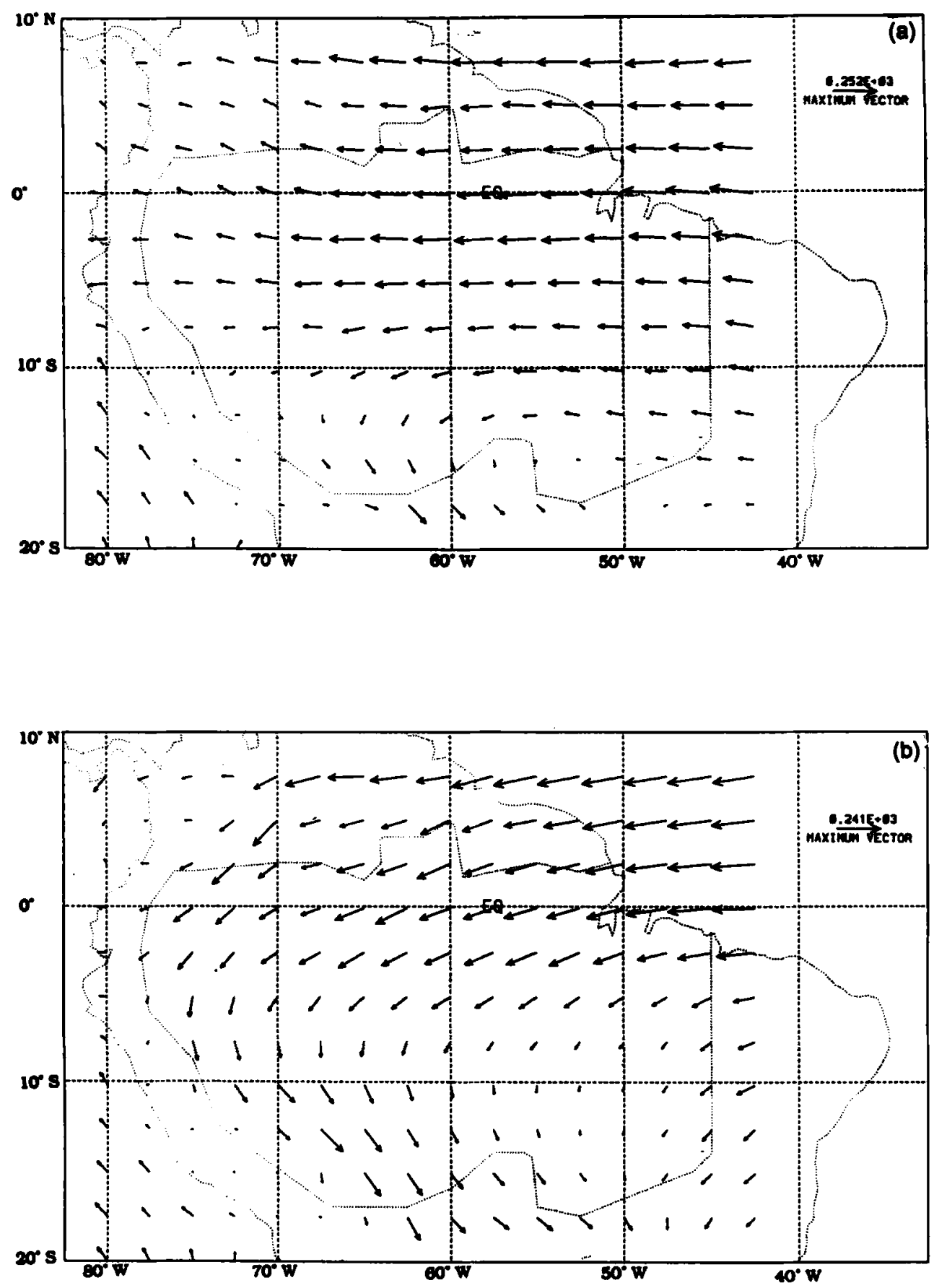

Figure 7. Distribution of water vapour flux over the Amazon basin, in $\mathrm{km}^{3}$ for: (a) June and (b) December. 

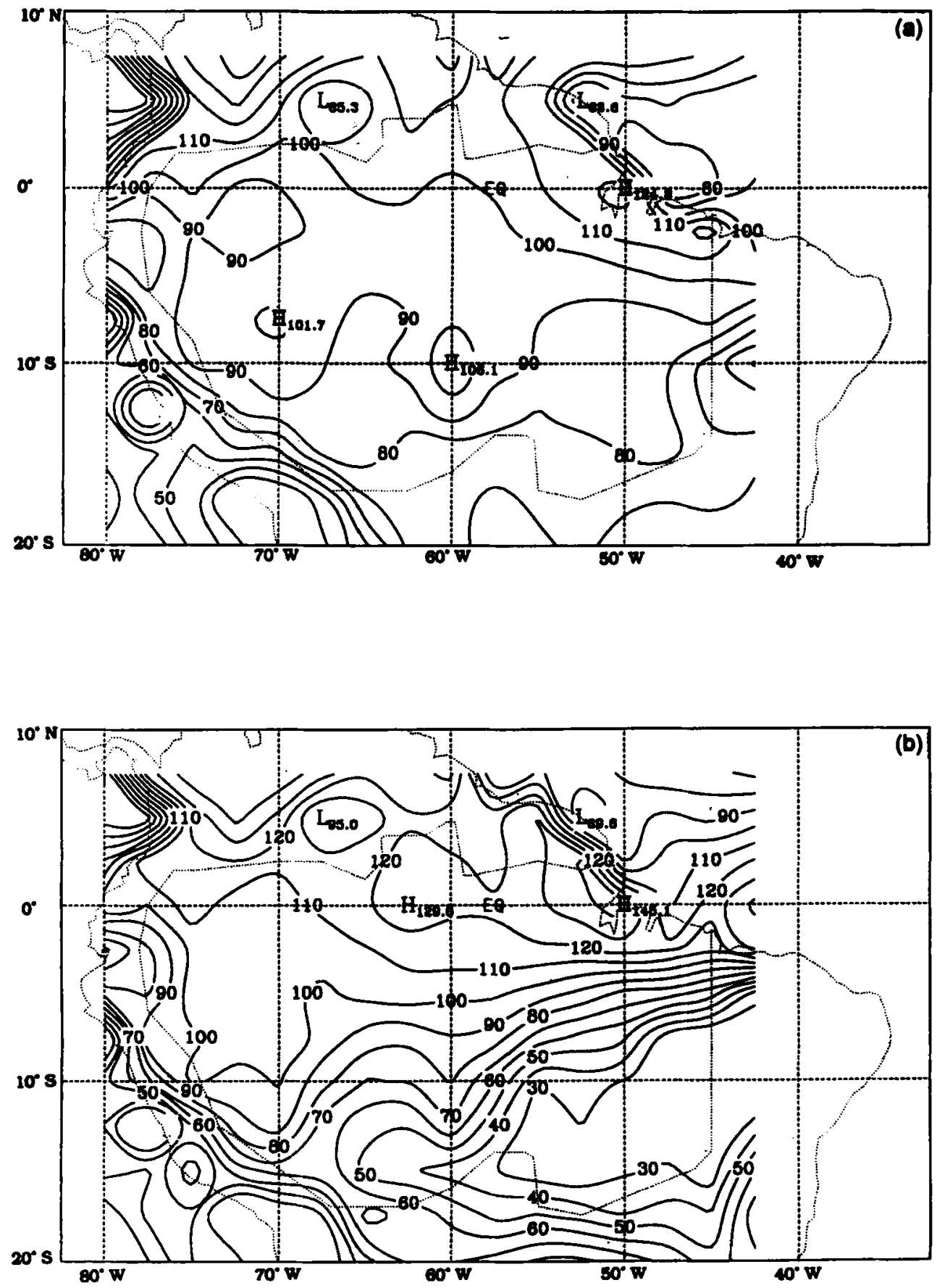

Figure 8. Evaporation in the Amazon basin in mm per month for: (a) June and (b) August. 

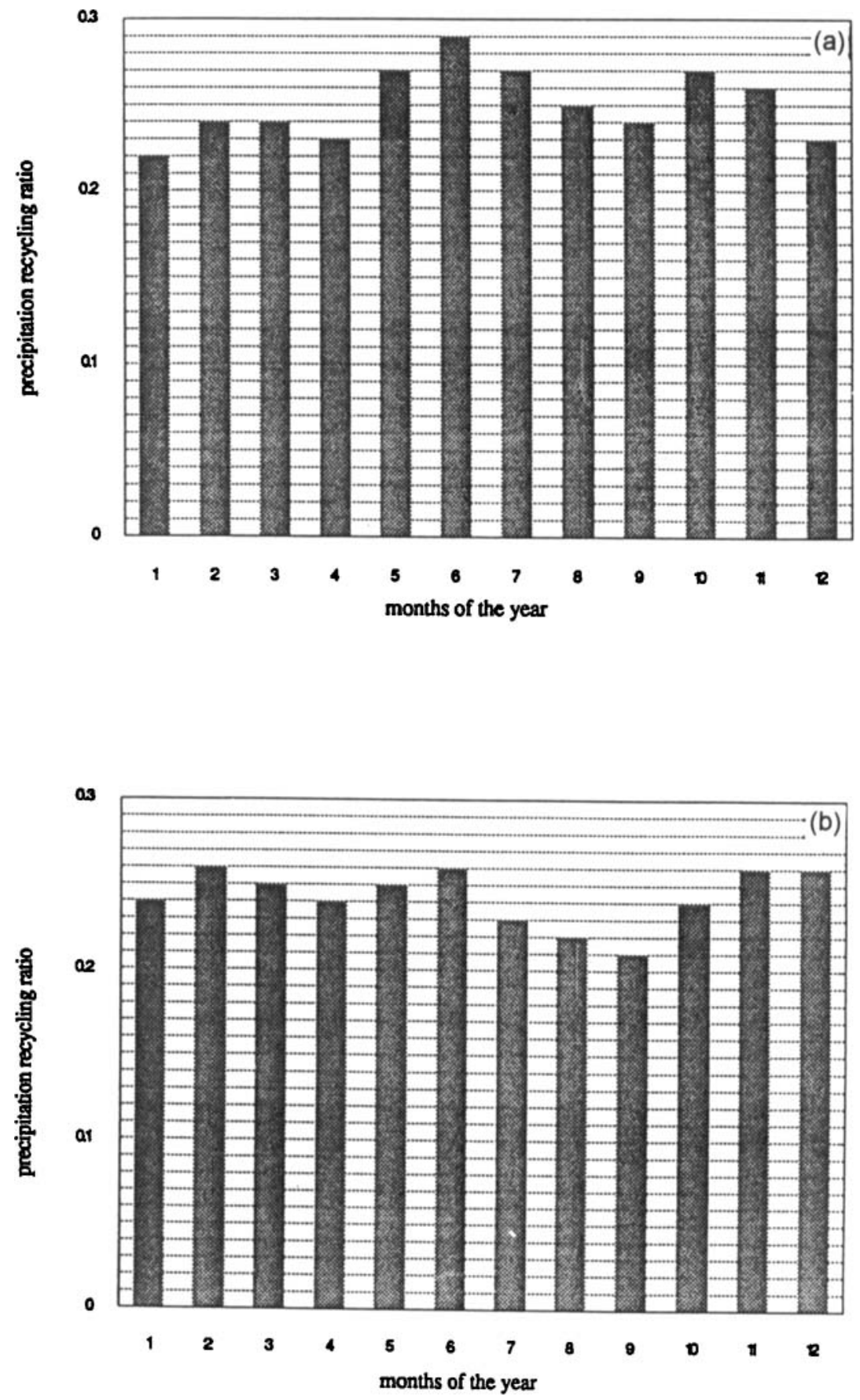

Figure 9. Seasonal variability of the precipitation recycling ratio in the Amazon basin: (a) applying Eq. (6) to each month's recycling map and (b) assuming a uniform spatial precipitation distribution. 


\section{ESTIMATION OF PRECIPITATION RECYCLING USING THE GFDL DATA SET}

The estimation procedure discussed above is applied to a different data set provided by the GFDL of the National Oceanographic and Atmospheric Administration, USA. The comparison of the estimates computed using the two different data sets provides a measure of the error bars around our estimates of recycling ratio in the Amazon basin. This comparison is necessary given the limited observational coverage in the Amazon region.

In this analysis the wind and humidity data are part of the GFDL data set. The evaporation data are part of the ECMWF data set, the same data used in section 5 (evaporation data have been validated independently in section 4). The GFDL data set consists of ten years (1963-73) of radiosonde observations. It includes daily measurements for the period 1963-68, and twice-daily measurements for the period 1968-73. The data are interpolated onto a regular grid by means of an objective analysis scheme. The resolution of the data is $2.5^{\circ}$ latitude by $5^{\circ}$ longitude. It is provided at 11 pressure levels between the surface and the tropopause. More information about the GFDL data can be found in Oort (1983).

The recycling model is applied to obtain estimates of the recycling ratio, following a similar procedure to that described in section 5 . It is found that $35 \%$ of precipitation in the Amazon basin is contributed by evaporation within the basin. This ratio is larger than the estimate of $25 \%$ which is obtained using the ECMWF data. Differences of similar magnitudes are found in the estimates of seasonal and spatial distributions of the recycling ratio. The difference between the two estimates provides a measure of the error bars around our estimates of the recycling ratio; those errors are in the order of $10 \%$ of total precipitation.

\section{WHY RECYCLING IN THE AMAZON BASIN IS OVERESTIMATED BY PREVIOUS STUDIES}

The studies of Molion (1975) and Marques et al. (1977) estimate that about $50 \%$ of precipitation in the Amazon basin is contributed by evaporation within the basin. These estimates are significantly larger than our estimates of recycling. The topography in the Amazon basin is such that the plain land is surrounded by mountains and high lands from the north, west and south. It is only open to the east where most of the atmospheric moisture enters the region from the Atlantic Ocean. This unique topographical setting may suggest that the atmosphere above the basin is a closed system. If that is true then the hydrologic cycle in the basin can be described by Fig. 10. In fact both of the studies mentioned above implicitly assume a hydrologic cycle similar to that in Fig. 10.

The atmospheric moisture flux into the region in Fig. 10 is finite, while the flux out of the region is zero; hence all the evaporation within the region precipitates. For the hydrologic cycle of Fig. 10 the ratio of recycled precipitation to total precipitation is equivalent to the ratio of total evaporation to total precipitation. Oltman (1967) estimates that the ratio of evaporation to precipitation in the Amazon basin is about $56 \%$, and based on this estimate Molion (1975) suggests that precipitation recycling in the Amazon basin accounts for about $56 \%$ of total precipitation. In a different study, Marques et al. (1977) consider the region between Belem and Manaus and estimate that the recycling ratio in this region is $48 \%$; this ratio is also equivalent to the ratio of evaporation to precipitation in that region. Hence, the accuracy of these estimates of recycling depends on the assumption that the outflow from the Amazon basin is negligible, i.e. the Amazon basin represents a closed system open only to inflow from the Atlantic Ocean. 


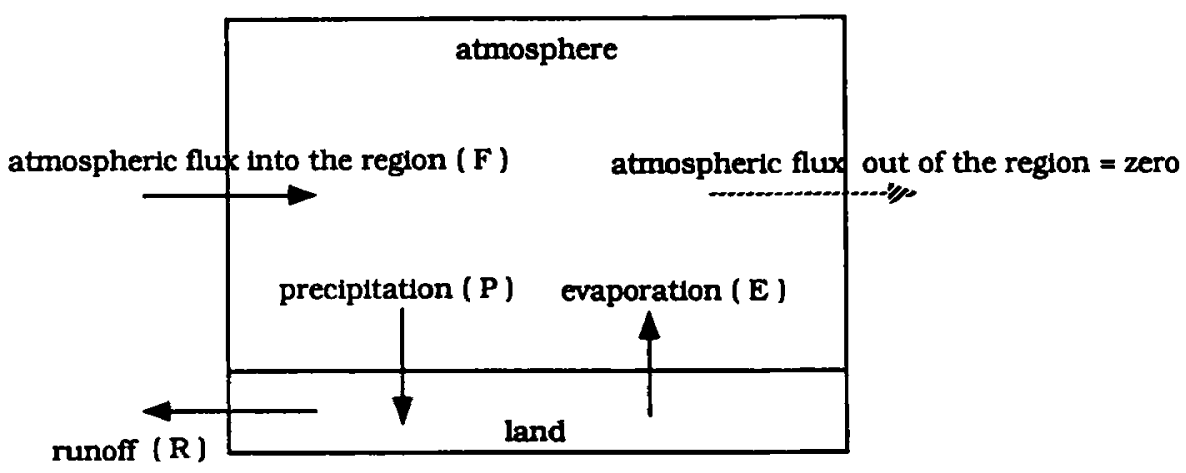

Figure 10. The hydrologic cycle of a closed atmospheric system.

The hypothesis that the atmosphere above the Amazon basin is a closed system is disproved by two observations. First, the isotopic data of Salati et al. (1979) suggests significant migration of atmospheric moisture out of the basin. The second evidence is given by the data in Fig. 11. It shows atmospheric moisture flux over the Amazon basin estimated from the ECMWF data set. The flux out of the basin accounts for about $68 \%$ of the flux into the basin, which indicates that the atmosphere above the Amazon is far from being a closed system, and indeed Fig. 10 is an inaccurate description of the hydrologic cycle in the Amazon basin. Hence, the estimates of the recycling ratio mentioned above represent an overestimate.

\section{DisCUSSION AND CONCLUSIONS}

The estimates of about 25 to $35 \%$ for the recycling ratio in the Amazon basin indicate a significant potential for interactions between surface hydrology and climate. Based on the results of this study using the EMCWF data the hydrologic cycle in the Amazon basin is described in Fig. 12. The fluxes of atmospheric water vapour and the recycling ratio are computed from the ECMWF data for the six years. The same figure compares the hydrologic cycles of the Amazon basin and the Mississippi basin (from Benton et al. 1950). The recycling ratio in the Amazon basin is significantly higher than the recycling ratio in the Mississippi basin (0.25 compared with 0.1 ). Another interesting difference between the two basins is that the ratio of precipitation to atmospheric inflow of water vapour is significantly higher in the Amazon basin ( 0.71 compared with 0.21 ). These two comparisons reflect not only the difference in atmospheric dynamics and rainfallproducing mechanisms between the tropics and mid-latitudes, but also the differences of the topographic settings and the vegetational cover of the two basins. It is important to note that the hydrologic cycle in Fig. 12 shows that the surface run-off ratio for the Amazon basin is about $42 \%$. This ratio is quite close to the observations in Oltman (1967) of about $44 \%$, based on measurements of river flow. This comparison provides an independent validation of the data used in this study.

The scale of this study is the total area of the Amazon basin and the resolution is $2.5^{\circ} \times 2.5^{\circ}\left(2.5^{\circ} \times 5^{\circ}\right.$ for the GFDL data). The recycling ratio is used to quantify the contribution of evaporation from the total area of the Amazon basin to precipitation at any point in the same basin. This definition should not be confused with the contribution of evaporation within a $2.5^{\circ} \times 2.5^{\circ}$ grid-cell to precipitation in that same cell. The recycling estimate for any region depends on the spatial-scale considered; the estimate 


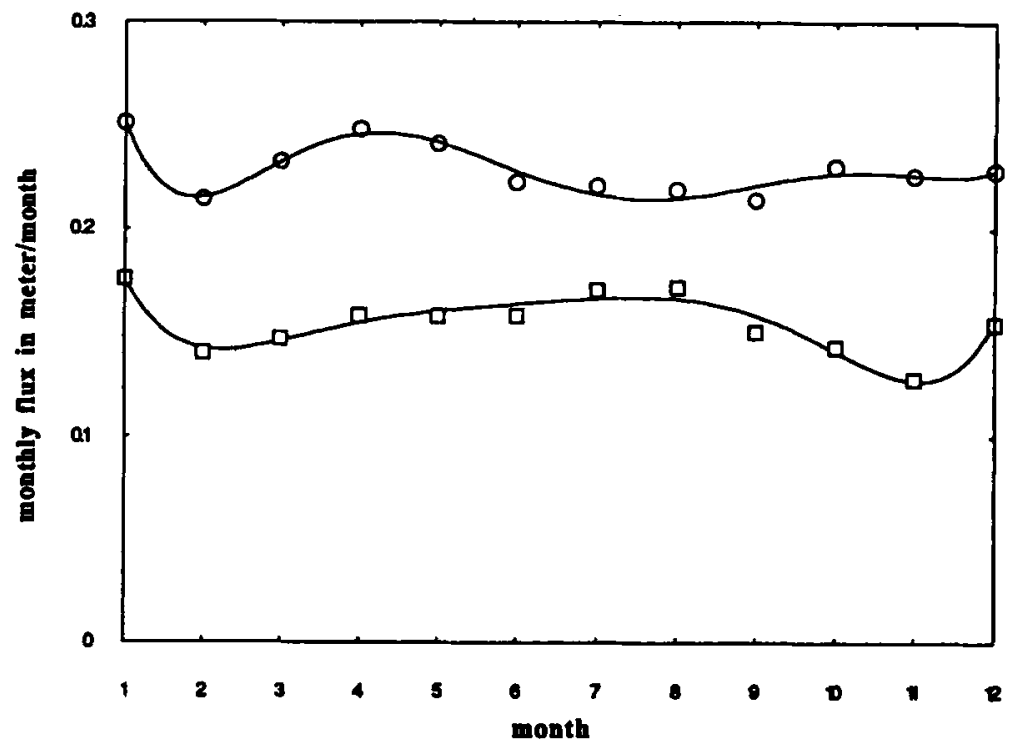

Figure 11. Water vapour flux at the boundaries of the Amazon basin: $O$ denotes flux into the basin and $\square$ flux out of the basin.
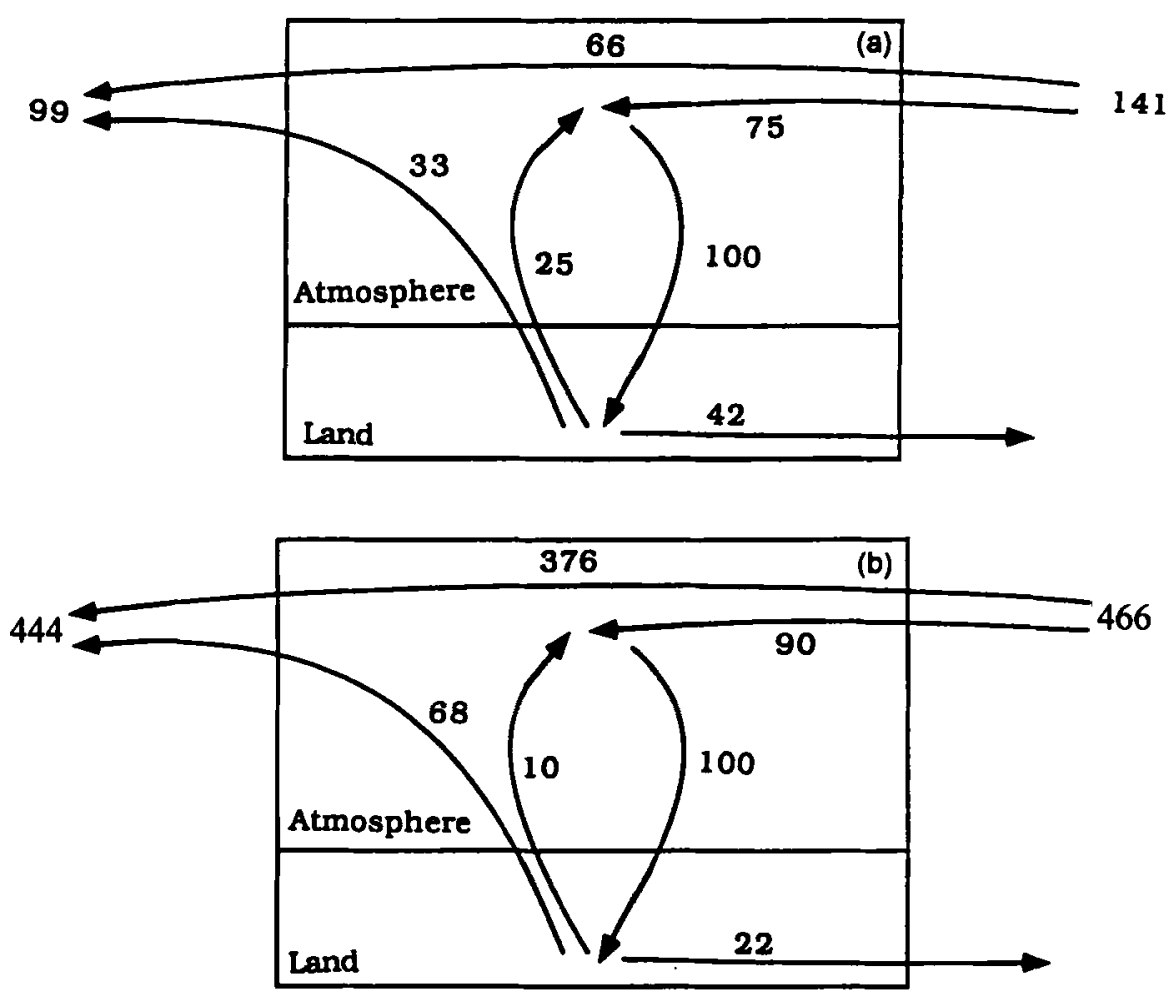

Figure 12. Hydrologic cycles: (a) the Amazon basin, annual precipitation $=1.95 \mathrm{~m}=100$ units; (b) the Mississippi basin, annual precipitation $=0.75 \mathrm{~m}=100$ units (estimates for the Mississippi basin are from Benton et al. (1950)). 
of $\rho$ would decrease with the size of the area considered. The estimate of recycling of $25 \%$ is not sensitive to the resolution used in this study for describing the spatial distribution of precipitation recycling. This sensitivity can easily be studied by considering the extreme case of treating the total area of the basin as one grid-cell. Using Eq. (6) with the data from Fig. 12(a) $\left(I_{\mathrm{w}}=0, I_{\mathrm{o}}=141, E=58\right) \rho$ is computed as $\rho=$ $(0+58) /(0+58+141)=0.29$. This estimate of $\rho$ is not significantly different from the conclusions of this paper, using $2.5^{\circ} \times 2.5^{\circ}$ as a resolution.

Precipitation in any region is directly proportional to the latent heat transported vertically from the boundary layer to the upper troposphere. The diabatic heating associated with condensation of water vapour is an important energy transport mechanism; it is strongly coupled to the dynamics of the tropical atmosphere (Gill 1980). The precipitation recycling ratio is a measure of how much of that energy is contributed by evaporation within the Amazon basin. Hence the results of this study indicate that $25 \%$ of the latent heat released over the Amazon basin is a result of recycling the net surface energy which is consumed in evaporation by the rain forest. The other $75 \%$ results from advecting energy from the Atlantic Ocean. The advected latent energy is part of the net surface energy that is consumed by evaporation from the ocean. This interpretation of the recycling results is important since it provides information about the degree of coupling between the atmospheres over the two adjacent regions.

The recycling maps presented in this paper reflect significant spatial and seasonal variability in recycling of water vapour in the Amazon basin. This variability is also reflected in the isotopic data of Salati et al. (1979). Under these conditions, the sensitivity of regional climate to changes in surface hydrology, e.g. deforestation, may very well depend on the location of the perturbation to surface hydrology.

The conclusion that the atmosphere above the Amazon basin is not a closed system suggests that outflow of atmospheric moisture from the basin may contribute important input to the hydrologic cycle in the surrounding regions. This is particularly important since it implies that changes in the Amazon basin evaporation, e.g. through deforestation, can potentially affect the moisture supply and rainfall in the surrounding regions.

It is important to emphasize that the estimates of precipitation recycling provide a diagnostic measure of the coupling between hydrology and climate. It would be inappropriate to make specific prognostic statements regarding the effects of deforestation, or any other disturbance of surface parameters, based solely on the estimates of precipitation recycling, and before studying the equilibrium of the disturbed climate system.

\section{ACKNOWLEDGEMENTS}

We thank Dr A. H. Oort for providing access to the GFDL data set. We acknowledge the contribution of the constructive criticism of two anonymous reviewers. We acknowledge the support of the National Aeronautics and Space Administration (NASA) (agreement NAG 5-1615). E. A. B. Eltahir is supported through a NASA graduate student fellowship (agreement NGT 30086). The views, opinions, and/or findings contained in this report are those of the authors and should not be construed as an official NASA position, policy, or decision, unless so designated by other documentation. 


\section{APPENDIX}

The monthly outflow of water vapour, $O$, and the change in storage of water vapour during a month, $\delta N$, are compared in Fig. 3. (Notice that inflow, $I$, and outflow, $O$, are of the same order of magnitude.) The data show that

$$
\delta N \ll O \text {. }
$$

But in deriving the model equations we are assuming that

$$
\delta N_{\mathrm{w}} \ll O_{\mathrm{w}}, \quad \delta N_{\mathrm{o}} \ll O_{\mathrm{o}} .
$$

In the following we show that the inequality (A.1) combined with the well mixed atmosphere assumption of Eq. (2) result in the inequality (A.2).

Since $\left(N=N_{\mathrm{w}}+N_{\mathrm{o}}\right)$ and $\left(O=O_{\mathrm{w}}+O_{\mathrm{o}}\right)$, Eq. (2) can be rewritten as

$$
\rho=N_{\mathrm{w}} / N=O_{\mathrm{w}} / O
$$

and since $\rho$ is assumed constant during any month Eq. (A.3) results in

$$
\begin{gathered}
\delta N_{w}=\rho \delta N \\
O_{w}=\rho O .
\end{gathered}
$$

By dividing Eq. (A.4a) by Eq. (A.4b) we get

$$
\delta N_{w} / O_{w}=\delta N / O
$$

Hence the observation that $\delta N \ll O$ combined with the well mixed atmosphere assumption implies that $\delta N_{w} \ll O_{w}$. Similarly it can be shown that the observation $\delta N \ll O$ combined with the well mixed atmosphere assumption implies that $\delta N_{0} \ll O_{0}$.

Benton, G. S., Blackburn, R. T. and 1950 Snead V. W.

Brubaker, K. L., Entekhabi, D. and 1991 Eagleson, P. S.

Budyko, M. I.

1974

Crum, T. D. and Stull, R. B.

1987

Dickinson, R. E. and Henderson-Sellers, A.

Gill, A. E.

Harris, R. C., Wofsy, S. C., Garstang, M., Browell, E. V., Molion, L. C. B.,

McNeal, R. J., Hoell Jr., J. M., Bendura, R. J., Beck, S. M., Navaro, R. L., Riley, J. T. and Snell, R. L.

Holzman, B.

Horton, R. E.

Illari, L.

1989

1988

1980

1988

\section{REFERENCES}

The role of the atmosphere in the hydrologic cycle. Trans. Am. Geophys. Union, 31, 61-73

'Atmospheric water vapor transport: estimation of continental precipitation recycling and parameterization of a simple climate model'. Parsons Laboratory technical report No. 333. Massachusetts Institute of Technology, Cambridge, Mass.

Climate and life. International Geophysical Series, vol. 18, Academic Press

Field measurements of the amount of surface layer air versus height in the entrainment zone. J. Atmos. Sci., 44, 27432753

Modelling tropical deforestation: A study of GCM land surface parametrizations. Q.J. R. Meteorol. Soc., 114, 439-462

Some simple solutions for heat-induced tropical circulation. Q. J. R. Meteorol. Soc., 106, 447-462

The Amazon Boundary Layer Experiment (ABLE 2A) dry season 1985. J. Geophys. Res., 93 (D2), 1351-1360

'Sources of moisture for precipitation in the United States'. U.S. Department of Agriculture Tech. Bull. No. 589

Hydrologic interrelations between lands and oceans. Trans. Am. Geophys. Union, 24, 753-764

The quality of satellite PWC data and their impact on analyzed moisture fields. Tellus, 41A, 319-337 
Lamb, R. G.

Lean, J. and Warrilow, D. A.

1989

Lettau, H., Lettau, $\mathbf{K}$. and Molion, L. C. B.

Marques, J., Santos, M., Villa Nova, N. A. and Salati, E. McDonald, J. E.

McNish, A. G.

Molion, L. C. B.

Oltman, R. E.

Oort, A. H.

Paluch, I. R.

Salati E., Dall'Olio, A., Matsui, E. 1979 and Gat, J. R.

Shukla, J., Norbe, C. and Sellers, P. 1990

Shuttleworth, W. J.

1988

Trenberth, K. E. and Olson, J. G.
1982 Diffusion in the convective boundary layer. Pp. 159-229 in Atmospheric turbulence and air pollution modelling. Eds F. T. M. Nieuwestadt and H. van Dop. Reidel Publishing Company

Simulation of the regional climatic impact of Amazon deforestation. Nature, 342, 411-413

1979 Amazonia's hydrologic cycle and the role of atmospheric recycling in assessing deforestation effects. Mon. Weather Rev., $107,227-238$

1977 Precipitable water and water vapor flux between Belem and Manaus. Acta Amazonica, 7, 355-362

The evaporation precipitation fallacy. Weather, 17, 168-177

Statistical aspects of long-range weather forecasting. Trans. Am. Geophys. Union, 1, 124-129

'A climatonomic study of the energy and moisture fluxes of the Amazon's basin with considerations of deforestation effects'. Ph.D. thesis, University of Wisconsin, Madison

Reconnaissance investigation of the discharge and water quality of the Amazon. Biota Amazonica, 3, 163-185

Global atmospheric statistics, 1958-1973. NOAA Prof. Paper No. 14, Washington, D.C.: U.S. Government Printing Office

The entrainment mechanism in Colorado cumuli. J. Atmos. Sci., 36, 2467-2478

Recycling of water in the Amazon basin: an isotopic study. Water Resour. Res., 15, 1250-1258

Amazon deforestation and climate change. Science, 247, 13221325

Evaporation from Amazonian rainforest. Philos. Trans. $R$. Soc. London, B, 233, 321-346

1988 ECMWF global analyses 1979-1986: circulation statistics and data evaluation. NCAR Technical Note NCAR/TN$300+$ STR 